Chapter 10

\title{
Traumatic Arteriovenous Fistula
}

\author{
Grace Carvajal Mulatti, André Brito Queiroz and \\ Erasmo Simão da Silva
}

Additional information is available at the end of the chapter

http://dx.doi.org/10.5772/56368

\section{Introduction}

Vascular injuries caused by civilian or war trauma represent a surgical challenge. These lesions are potentially lethal and can cause death at the scene. One of the most fascinating and misdiagnosed complications of vascular injuries is the arteriovenous fistula (AVF), which results from a direct communication between an artery and a vein. They are usually secondary to penetrating trauma and occasionally may be diagnosed many years after the injury.

\section{Historic data}

Knowledge and experience on vascular trauma was obtained especially in wartime. In 1757 William Hunter has described the physiopathology associated with an AVF and since then the management of these clinical problem has challenged many surgeons.

In addition to case reports of the $19^{\text {th }}$ Century, there have been many published series resulting from wartime experience throughout the $20^{\text {th }}$ Century. Much of the experience acquired from treating and diagnosing AVF comes from the II World War and the Korean and Vietnam Wars. In the Korean conflict as many as 215 fistulas and aneurysms have been described. Cumulative, fistulas and false aneurysms may account for $7 \%$ of the casualties in Vietnam. At that time, diagnosis was established by physical examination with palpation and auscultation, and confirmed by arteriography.

Most cases of arteriovenous fistulas and pseudoaneurysms were treated conservatively in wartime, and operated only later.

Most recently, experience has been obtained from the Iraq and Afghanistan wars. Treatment has evolved over the past decades thanks to the endovascular techniques, however early 
diagnosing still seems to be a challenge. Even in recent studies arteriovenous fistulas are frequently occult findings and are diagnosed only when an arteriogram is performed.

\section{Etiology}

Traumatic AVFs are usually caused by penetrating trauma, accounting for as many as $90 \%$ of cases. Blunt trauma is rarely the cause and it is responsible for the other $10 \%$. Gunshot wounds are the most frequent ones, but stab wounds and iatrogenic lesions may also account for traumatic AVFs.

Most of the military trauma injuries affect the extremities. On the other hand extremities and abdominal injuries are equally distributed in the civilian population.

Traumatic AVFs often result from a cold weapon or a small-caliber bullet, as high-velocity lesions are usually cause of hemorrhage and potentially hemodynamic shock (Fig 1 and 2).

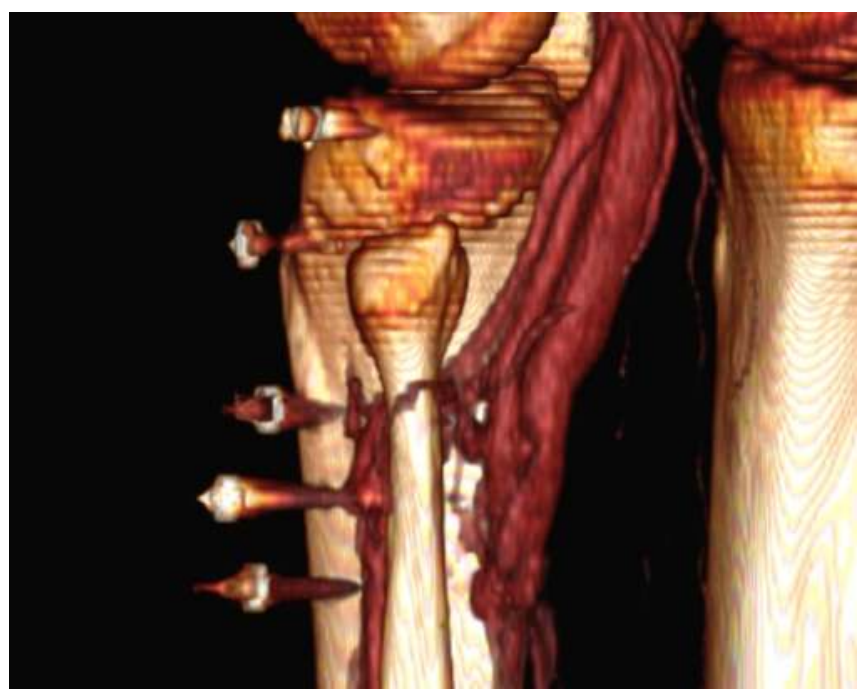

Figure 1. Three-dimensional computed tomography $(\mathrm{CT})$ reconstruction shows an AVF involving below the the knee vessels and multiple bullet shrapnel near the lesion.

When it comes from iatrogenic lesions, the most frequent are the ones caused by percutaneous interventions such as renal biopsies, cardiac catheterization and orthopedic procedures. Therapeutic procedures and their complexity increase the risk. Following cardiac catheterization the femoral AVF is the most common. Nonetheless it has also been reported carotid or subclavian AVFs following placement of a central venous catheters. 


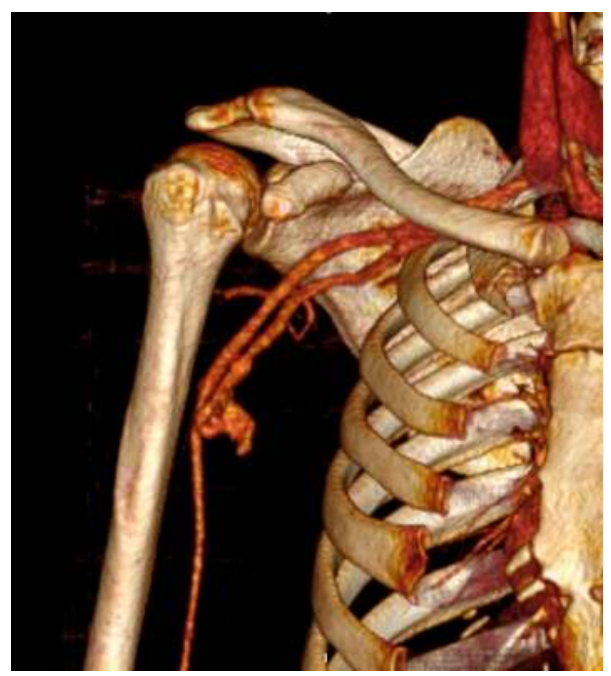

Figure 2. Three dimensional CT reconstruction shows a brachial AVF associated with a pseudoaneurysm after a stab wound in the arm.

Carotid-cavernous fistulae (CCF) are usually traumatic and potentially lethal. Mechanisms include fractures to the base of the skull, penetrating wounds and even more rarely rupture of preexisting aneurysms.

\section{Clinical signs}

Post-traumatic AVF is an unusual disease with a wide variability of presentations. They are often asymptomatic, but when significant can cause rapid shunting with return of oxygenated blood to the right heart. Clinical signs may be detected from a few hours to many years after the injury and may vary according to the location and diameter of the affected vessels.

The clinical diagnosis of a traumatic AVF is based on a history of trauma and meticulous physical examination. Physical examination with palpation and auscultation may reveal thrills, murmurs, bruits and a pulsatile mass. Distal pulses may be palpable, but the AVF can shunt away blood from the extremity and cause symptoms of distal ischemia.

The Nicoladoni-Israel-Branham's sign may be present: the manual compression of the fistula or the artery proximal to the fistula causes a decrease in the heart rate. This maneuver produces an increase in peripheral vascular resistance and afterload. The response to the increased afterload is a reflex bradycardia.

In a trauma scenario bone fractures or neurologic deficits can raise the suspicion of a vascular injury, especially when the physical examination is not remarkable. 
Chronic fistulas are even more rare, and large flow AVFs can present with symptoms of high output congestive heart failure, edema and cardiomegaly. Those more prompt to develop a delayed presentation are young trauma victims.

Aneurysmal degeneration of the involved arterial and venous segments may also occur due to structural changes secondary to hemodynamic stress (Fig 3). Fistulas affecting the extremities may lead to signs of venous hypertension, varicosities, pain and limb overgrowth. Pulsatile varicose veins can draw attention to the pathology lying underneath (Fig 4 and 5).

As regard to CCF, clinical signs include pulsatile exophthalmos, eyeball redness, bruit, diplopia, ptosis, visual disturbances, and loss of visual acuity. During physical examination it has been observed that the bruit shall disappear should the examiner occlude the ICA by digital compression.

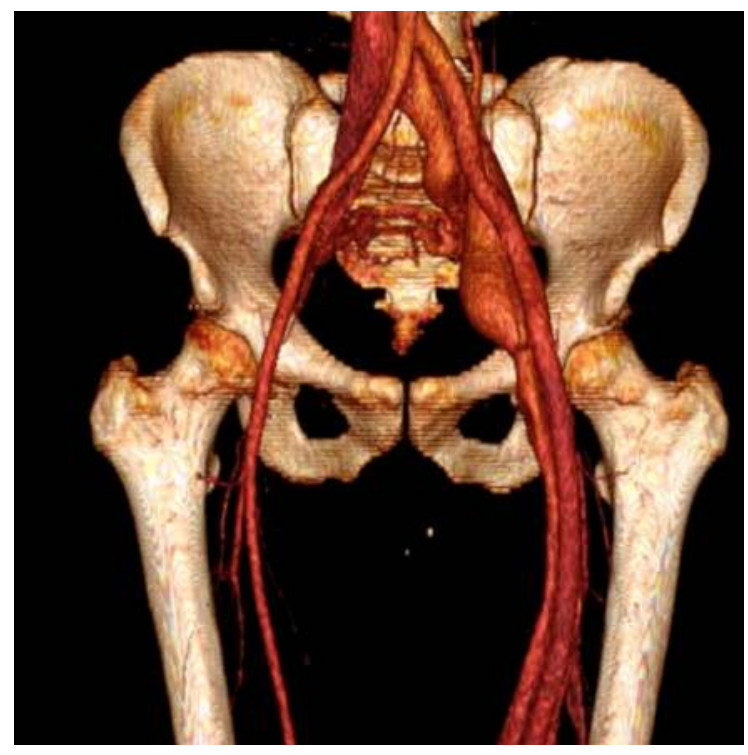

Figure 3. Three-dimensional CT reconstruction shows aneurysmal degeneration of iliac and femoral arteries and veins secondary to an AVF involving below the knee vessels.

According to literature, the most frequent locations are the femoral and popliteal vessels. Other sites include aorto-caval, iliac, carotid-jugular and renal fistulas.

The differential diagnosis must include pseudoaneurysm, true aneurysm, arteriovenous malformation, cyst, abscess and hematoma. One must remember that nonvascular lesions may appear pulsatile due to transmitted pulsation from adjacent arteries. 


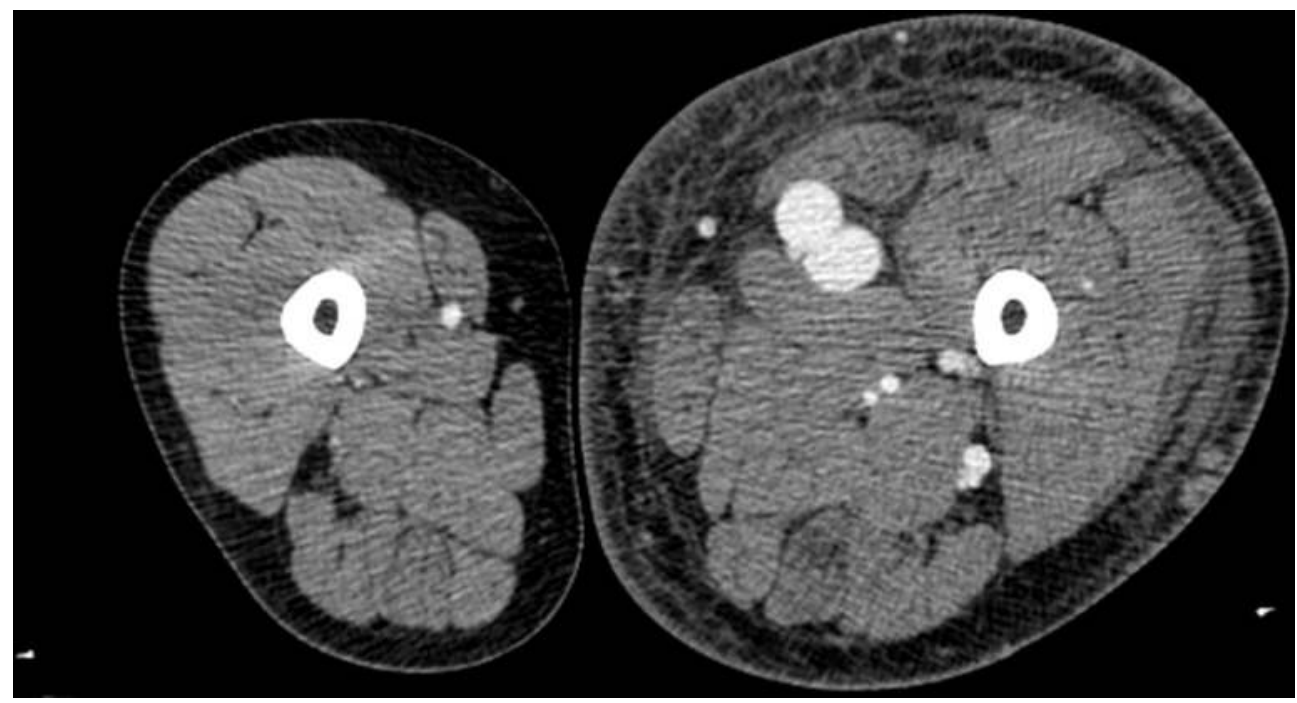

Figure 4. Contrast-enhanced CT demonstrates an AVF between femoral artery and vein, secondary to a gunshot wound 15 years earlier, with left lower limb overgrowth and venous hypertension.

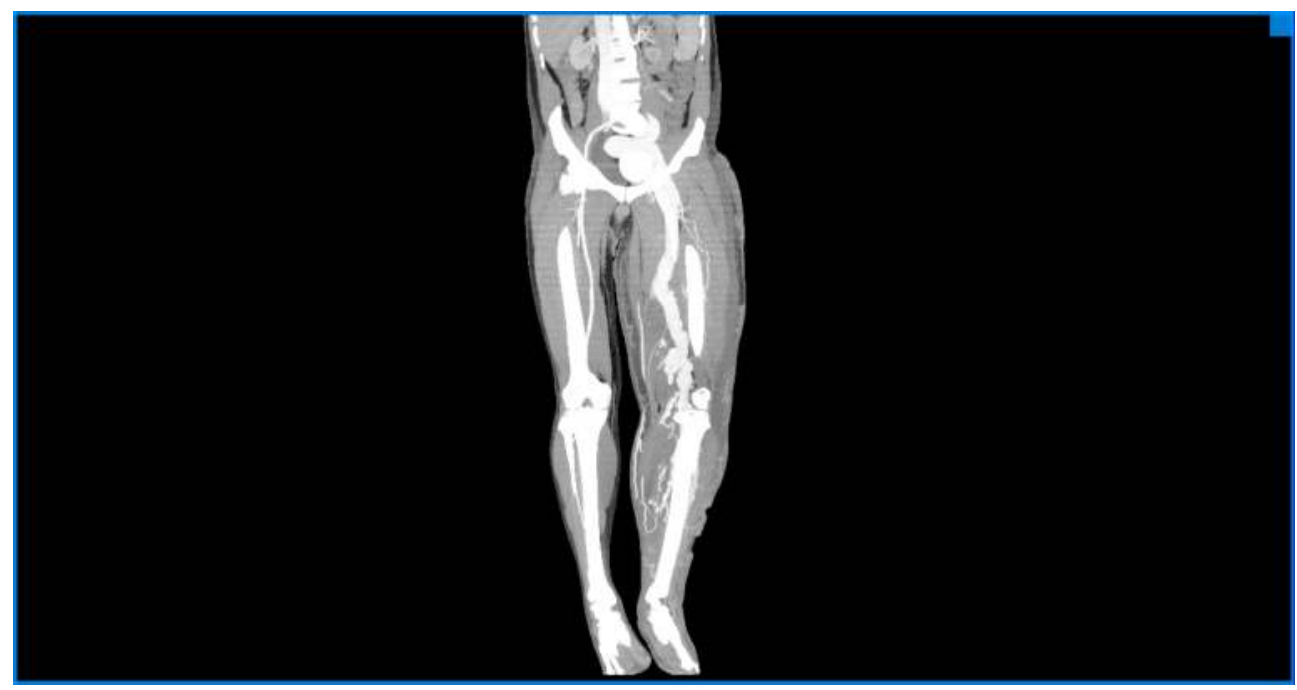

Figure 5. The same patient of figure 4, computed tomography in coronal view demonstrates left lower limb overgrowth and dilated iliac and femoral vessels. 


\section{Diagnostic imaging}

Diagnosis is usually difficult and greatly dependent on imaging studies. Arteriography was the main exam in the 1960 and 1970s and still plays an important role in diagnosing AVF. However, nowadays the computed tomography (CT) angiography, magnetic resonance imaging (MRI) and color Doppler have been playing an important role in the diagnosis of this pathology.

Arteriography is still the gold standard exam for diagnosing vascular traumas, including traumatic AVFs. Catheter based angiography is a dynamic study and can, in most cases accurately demonstrates the arteries filling the fistula and the exact point of the arteriovenous communication. The major disadvantages of conventional arteriography are the cost of the procedure, the delay that occurs before arteriography, and the need for a specialized team comprising a physician, angiography technologist, and nurse. Besides being an invasive exam that requires an arterial access it can result in a number of different complications.

Computed tomographic angiography is a reliable and convenient imaging modality for diagnosing AVF after blunt and penetrating trauma. It is a noninvasive modality that could replace conventional arteriography as the initial diagnostic study for arterial injuries after trauma, even when the suspected diagnosis is an AVF. The technique requires scanning with multidetector helical CT after rapid intravenous injection of iodinated contrast material. This is a noninvasive, accurate, and easily accessible diagnostic imaging test acquired by a single trained technologist.

In recent years CT is increasingly available in the emergency setting. The development of helical CT scanners with a multi-detector row configuration and three-dimensional postprocessing has further encouraged the use of CT angiography in the evaluation of a suspected vascular injury. High quality axial images and reformatted angiography exams can be obtained in a shorter time than with other modalities and with a lesser complication rate than catheter angiography.

In some cases metallic streak artifact, motion artifact, and inadequate arterial opacification may render a CT angiogram nondiagnostic. Streak artifacts are the major limiting factor of CT angiography to demonstrate AVFs after penetrating missile injuries. The deposited metallic fragments may create streak artifacts that obscure the arteries, commonly at the exact location where the AVF is likely to occur (Fig. 6). In these cases, angiography is necessary for optimal assessment.

Contrast-enhanced CT has also an important role in surgical planning because it can reveal where the lesion is, its extension, and its relation to adjacent structures, mainly in the abdominal or thoracic cavities. It is also important for endovascular treatment planning allowing precise measurements of the diameters and length of involved vessels. Computed tomography and MRI typically show early contrast filling in the vein during the arterial phase (Fig. 7). 


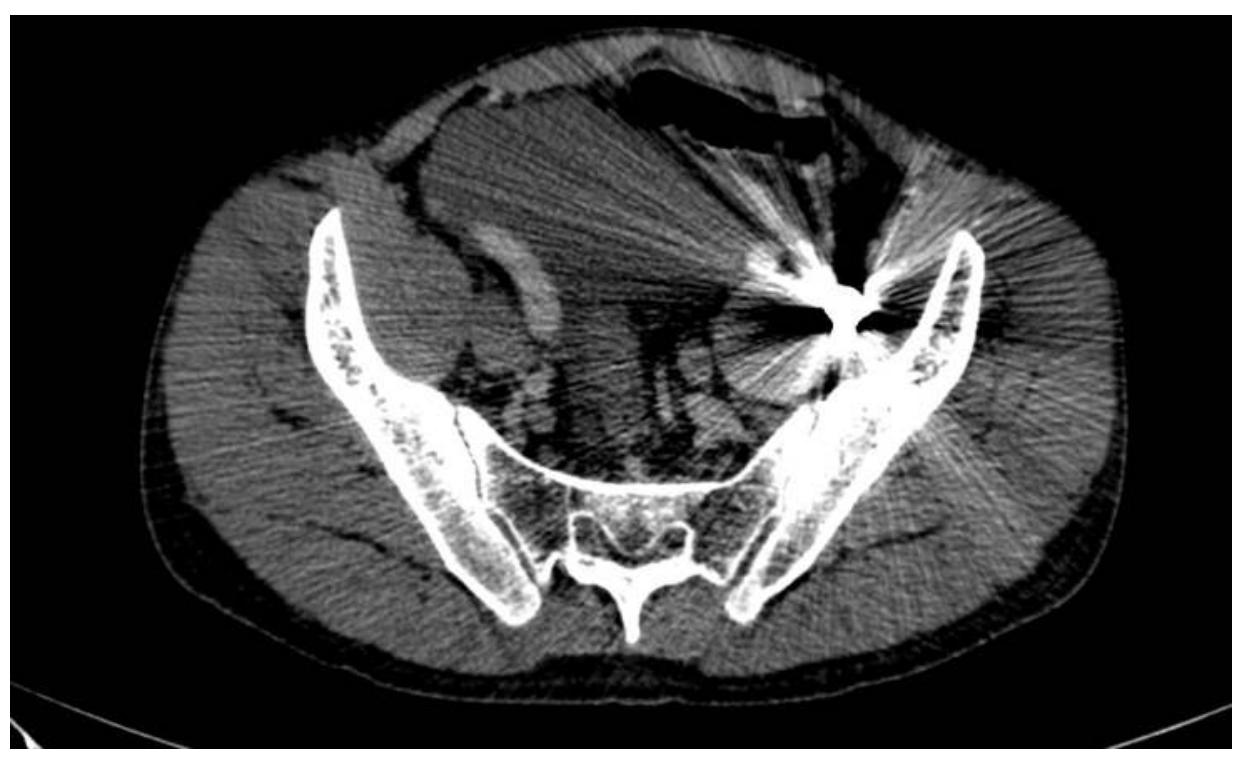

Figure 6. Computed tomography in a patient who sustained an iliac AVF with bullet fragments obscuring the vessels near the arteriovenous communication

Angiography and CT share the same complications rates related to the use of iodinated contrast, like allergic reactions and renal function impairment and requires also the use of radiation for execution.

Some authors have also proposed MRI for assessment of vascular injuries including traumatic AVF, but it may be limited for depiction of concurrent osseous injuries. Furthermore, MR is not well suited for some patients with impacted metallic fragments and acutely injured ones requiring life support devices as they may be not compatible with the magnet.

In chronic AVF both the artery and the vein dilate and elongate in response to the greater blood flow and shear stress, but the vein dilates more and becomes "arterialized". Findings include a pseudoaneurysm, large venous aneurysms, proximal dilation of the artery or hematoma of the vessel wall (Fig. 7).

In the setting of a suspected AVF, not confirmed by physical findings, the color doppler, a simple non-invasive method can usually clear out the diagnosis. Gray-scale ultrasound imaging is not helpful in the evaluation of acute AVF, although may be important in chronic AVF when the high flow state has caused dilatation of the vein and artery. Color doppler imaging is usually diagnostic. Tissue vibrations caused by turbulent flow are the most notable color Doppler finding. Also, the exact spot of the arteriovenous communication can sometimes be identified. The Doppler waveform in the feeding artery shows a low resistance pattern with increased diastolic flow. The jet of arterial flow entering the vein can cause a marked flow disturbance and chaotic waveform or an arterial waveform is present in more severe cases (Fig 8, 9). 


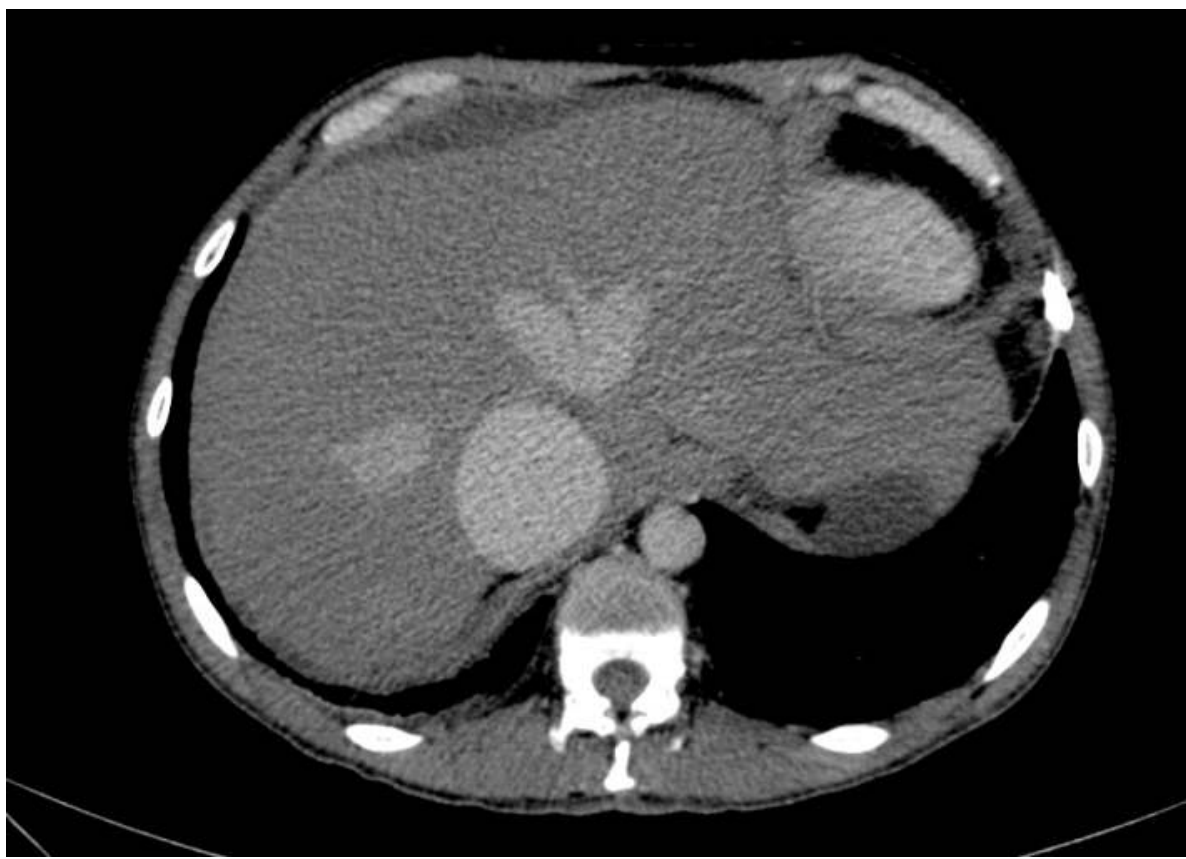

Figure 7. Contrast-enhanced CT shows dilated inferior vena cava with the same contrast opacification of the aorta in the arterial phase in a patient with iliac AVF.

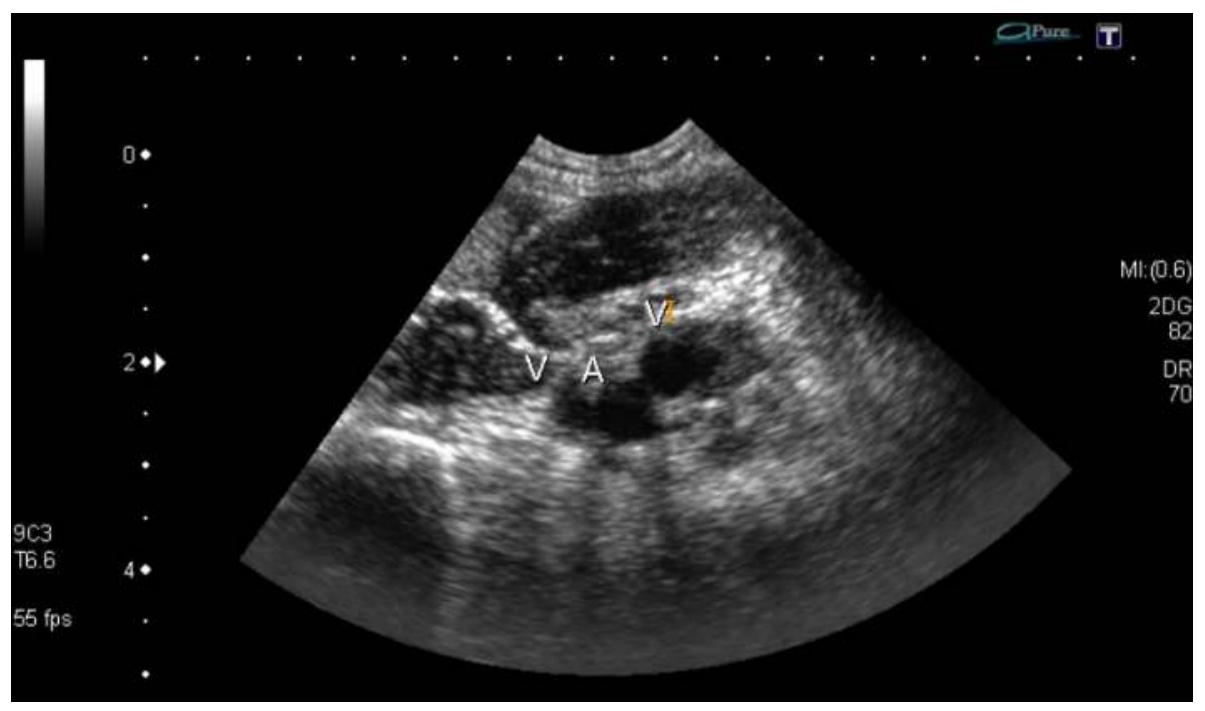

Figure 8. Ultrasound demonstrates the arteriovenous communication in a brachial AVF secondary to a stab wound. 


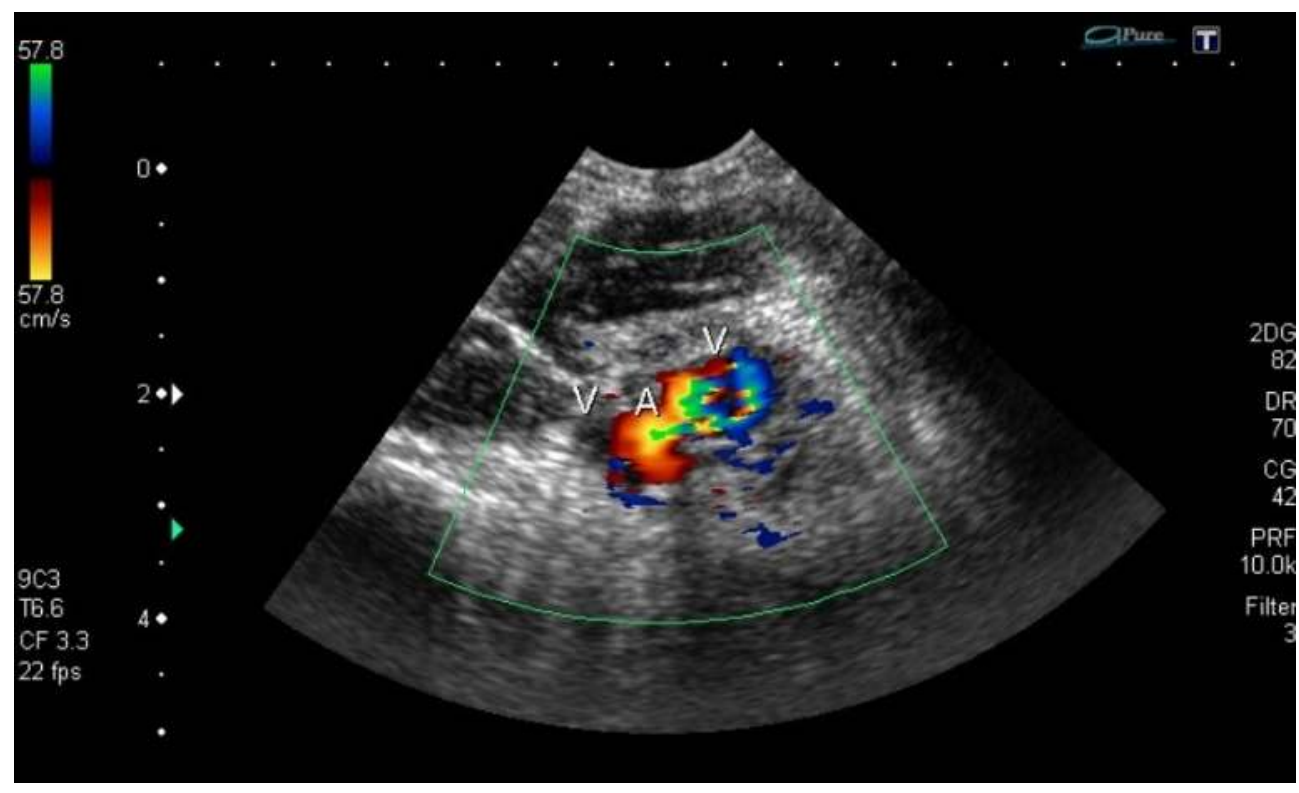

Figure 9. Color Doppler shows the communication between the brachial artery and vein with marked flow disturbance in the same patient of figure 2 .

All imaging studies used in the diagnosis of arterial or venous diseases may play a role in traumatic AVF diagnosing. Nowadays color doppler and CT angiography are the usual exams, and the catheter based angiography is performed only for the more complex cases and as confirmatory exam or when it is necessary for planning the endovascular treatment.

\section{Treatment}

Early recognition and prompt repair may lead to a significant decrease in the number of complications such as congestive heart failure or venous hypertension of the limb. AVFs do not resolve spontaneously in the majority cases. The aim of the treatment is the closure of the $\mathrm{AVF}$, with preservation of patency of the main vessels.

Surgical repair using traditional surgical techniques is feasible. However, an open approach for many of these lesions may be fraught with peril owing to grossly distorted and edematous tissue planes. Arterial and venous repair with the saphenous vein or prosthetic graft were largely used in the war series and are still used nowadays. Venous ligation can be used depending on its diameter, quadruple ligation-excision and complex reconstructions may be used. However, late presentation of AVF is prone to significant intraoperative bleeding due to the complex venous anatomy encountered during surgical dissection and repair. Associated morbidities can include limb ischemia, gangrene, limb loss, Vena Cava thrombosis, pulmonary embolus, venous stasis, and uncontrollable bleeding. 
In an effort to avoid the morbidity and mortality associated with an open approach for traumatic AVFs, the focus has shifted to endovascular techniques. The main advantage of endovascular approach is that the system can be inserted through a remote access site, obviating the need for extensive surgical exposure. Endovascular repair can include the use of covered stents and coil embolization, depending on the location and diameter of the involved vessels. In some cases open surgical repair has prohibitive morbidity and mortality, and a minimal invasive technique has emerged as an effective treatment alternative. Recognized advantages of endovascular repair for traumatic AVF compared with open repair include diminished pain, decreased disability and rapid recovery. The first recorded repair of an AVF using an endovascular approach was in 1992 by Parodi when a covered stent was deployed to treat a traumatic AVF in subclavian vessels.

The goal of endovascular therapy is the selective elimination of the vascular lesion, with the preservation of normal patency of the essential vessels. Endovascular therapy using covered stents and coils are both feasible and safe for treating traumatic AVF. Of these endovascular methods, stent-graft placement can be used to exclude the fistula from circulation and preserve the parent artery and vein. Embolization with coils may be used in selective cases, when nonessential vessels are responsible for the arteriovenous communication, and both procedures may be associated. Complications during covered stent placement are due to dissection or rupture and embolization of the devices causing ischemic complications.

Subacute thrombosis and intimal hyperplasia leading to in-stent stenosis or vessel occlusion are other complications associated with covered stents. The long-term patency of these devices is unknown. Some authors consider that balloon-expandable covered stents like Advanta V12 (Atrium, USA) are better when accurate deployment is required. We believe that newer selfexpanding devices, such as Fluency (Bard, USA) and Viabahn (Gore, USA), which are stent

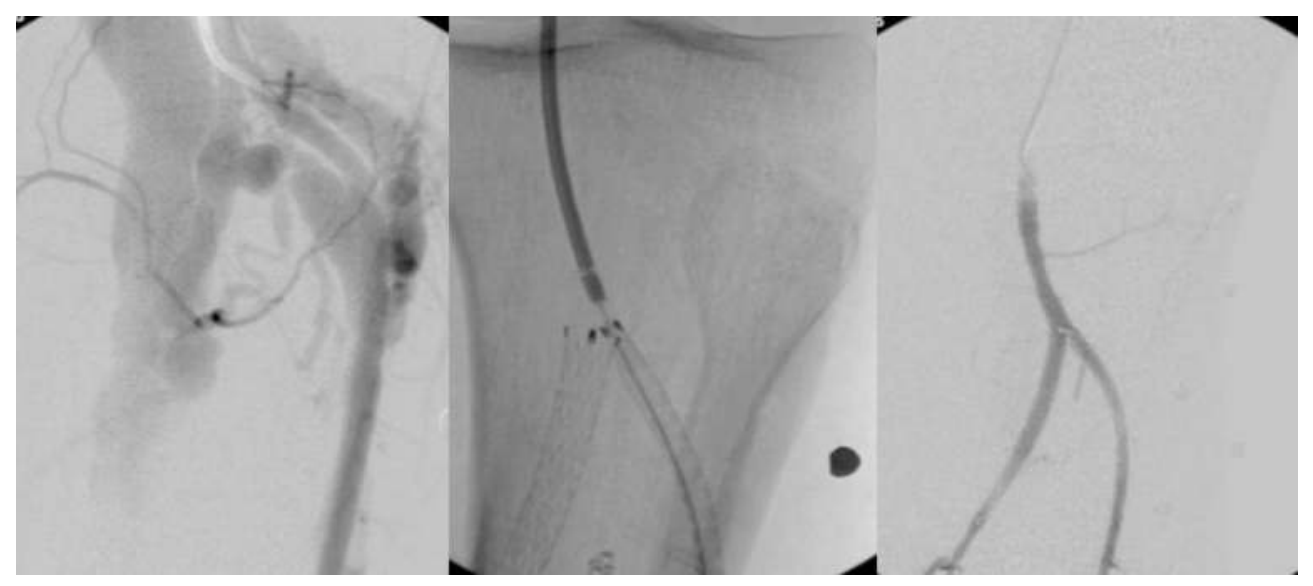

Figure 10. Sequential figures show catheter-based angiography demonstrating AVF in tibial anterior and posterior vessels, subsequent self-expanding covered stents deployment and final arteriography demonstrating closure of the arteriovenous communication. 
grafts composed of nitinol stents covered with polytetrafluoroethylene, are more flexible, conform more easily to the vessel walls, and the polytetrafluoroethylene covering is less thrombogenic. Therefore, we thought that these devices appear to be more suitable. When chronic AVF presents with very enlarged vessels the use of endografts primarily designed for aortic and iliac diseases may be used in aorto-iliac and other vessels (Fig. 10,11, 12).

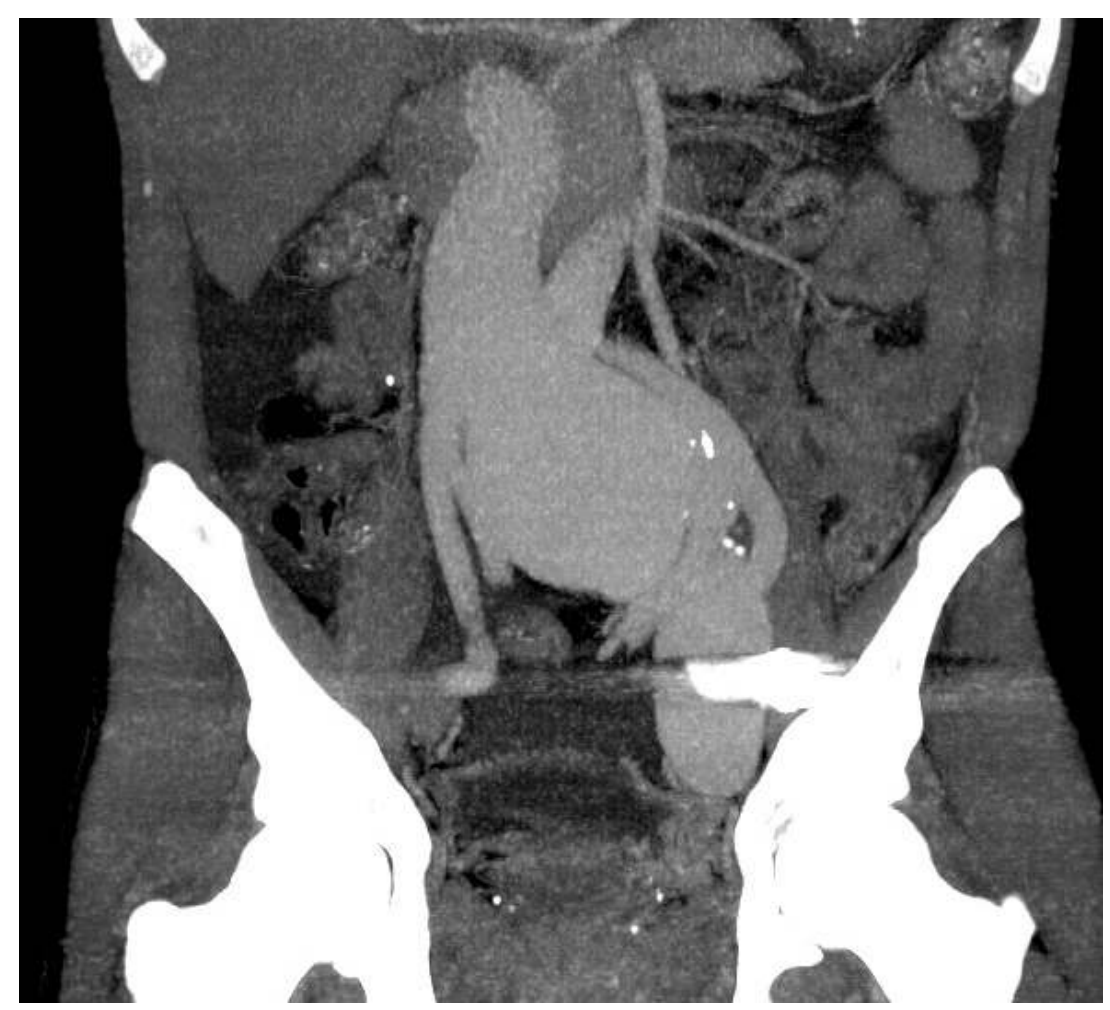

Figure 11. Computed tomography in coronal view show an iliac AVF dilated iliac veins and inferior vena cava, with the same contrast opacification.

Due to the reduced venous flow following AVF closure, venous thrombosis can occur especially when venous aneurysms are present, carrying out complications like pulmonary embolism. In this situation systemic anticoagulation is recommended for at least six months after the AVF treatment. Inferior vena cava filters may have significant importance in some cases, however attention to the diameter of these veins is essential to prevent migration of the filters.

Neuroradiologists or interventional radiologists usually carry out treatment of CCFs with innumerous endovascular possibilities, such as coil embolization, double balloon occlusion technique or occlusion of the ICA by use of detachable balloon, the last being the gold standard. 


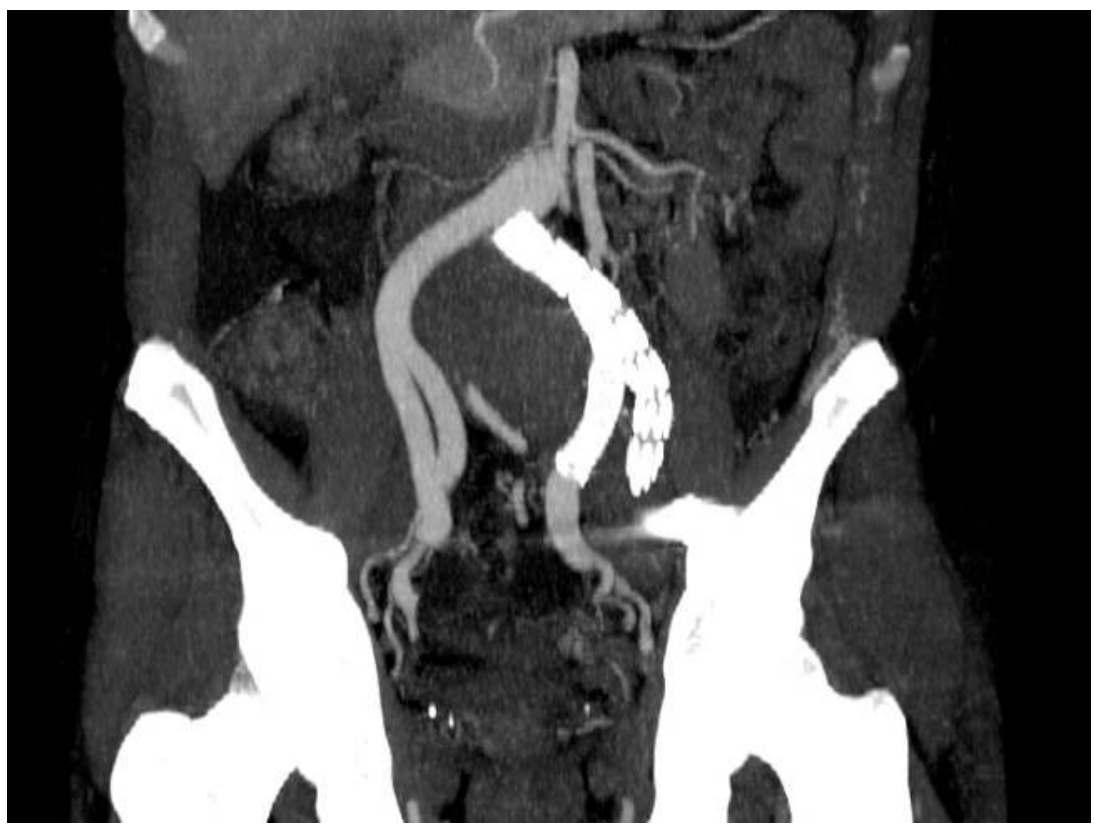

Figure 12. Computed tomography in coronal view of the same patient in figure 10 after endovascular treatment with covered devices demonstrates no venous opacification and pervious iliac arteries.

\section{Author details}

Grace Carvajal Mulatti, André Brito Queiroz and Erasmo Simão da Silva

Vascular and Endovascular Surgery Division, São Paulo University Medical School, São Paulo-SP, Brazil

\section{References}

[1] Anderson, C. A, \& Strumpf, R. K. Dietricht EB: Endovascular management of a large post-traumatic iliac arteriovenous fistula: Utilization of a septal occlusion device. J Vasc Surg (2008). , 48, 1597-9.

[2] Creech, O, \& Gantt, J. Wren H: Traumatic Arteriovenous Fistula at Unusual Sites. Ann Surg. (1965). June; , 161(6), 908-920.

[3] Davison, B. D, \& Polak, J. F. Arterial injuries: a sonographic approach. Radiol Clin North Am. (2004). Mar;" 42(2), 383-96. 
[4] DuBose JRecinos G, Teixeira PG, Inaba K, Demetriades D. Endovascular stenting for the treatment of traumatic internal carotid injuries: expanding experience. J Trauma. (2008). Dec;; 65(6), 1561-6.

[5] Fattahi, T. T, Brandt, T, Jenkins, W. S, \& Steinberg, B. Traumatic Carotid-Cavernous Fistula: Pathophysiology and Treatment. J Craniof Surg. (2003). Mar;, 14(2), 240-6.

[6] Faure, E, Canaud, L, Marty-ané, C, \& Alric, P. Endovascular repair of a left common carotid pseudoaneurysm associated with a jugular-carotid fistula after gunshot wound to the neck. Ann Vasc Surg. (2012). Nov;26(8):1129.e, 13-6.

[7] Faure, E, Canaud, L, \& Marty-ané, C. Alric P: endovascular Repair of a Left Common Carotid Pseudoaneurysm Associated With a Jugular-Carotid Fistula After Gunshot Wound to the Neck. Ann Surg, (2012). ee16., 13-1129.

[8] Feliciano, D. V, Bitondo, C. G, Mattox, K. L, et al. Civilian trauma in the 1980's. A 1year experience with 456 vascular and cardiac injuries. Ann Surg (1984). , 199, 717-723.

[9] Fox, C. J, Gillespie, D. L, \& Donnell, O. SD, et al: Contemporary management of wartime vascular trauma. J Vasc Surg (2005). , 41, 638-644.

[10] Huang, W, Villavicencio, J. L, \& Rich, N. M. Delayed treatment and late complications of a traumatic arteriovenous fistula. J Vasc Surg. (2005). Apr;, 41(4), 715-7.

[11] Hughes CW: Arterial repair during the Korean WarAnn Surg (1958). , 147, 555-561.

[12] Nagpal, K, Ahmed, K, \& Cuschieri, R. J. Diagnosis and management of acute traumatic arteriovenous fistula. Int J Angiol (2008). , 17(4), 214-216.

[13] Khoury, G, Sfeir, R, Nabbout, G, Jabbour-khoury, S, \& Fahl, M. Traumatic arteriovenous fistulae: "the Lebanese war experience". Eur J Vasc Surg. (1994). Mar;, 8(2), 171-3.

[14] Kim, J. W, Kim, S. J, \& Kim, M. R. Traumatic carotid-cavernous sinus fistula accompanying abducens nerve (VI) palsy in blowout fractures: missed diagnosis of "whiteeyed shunt". Int J Oral Maxillofac Surg. (2013). Apr;, 42(4), 470-3.

[15] Kollmeyer, K. R, Hunt, J. L, Ellman, B. A, \& Fry, W. J. Acute and chronic traumatic arteriovenous fistulae in civilians. Arch Surg (1981). , 116, 697-702.

[16] Kuhlencordt, P. J, Linsenmeyer, U, Rademacher, A, et al. Large external iliac vein aneurysm in a patient with a post-traumatic femoral arteriovenous fistula. J Vasc Surg (2008). , 47, 205-8.

[17] Miller-thomas, M. M, West, O. C, \& Cohen, A. M. Diagnosing traumatic arterial injury in the extremities with CT angiography: pearls and pitfalls. Radiographics. (2005). Oct;25 Suppl 1:S, 133-42.

[18] Parodi, J, \& Barone, H. Transluminal treatment of abdominal aortic aneurysms and peripheral arteriovenous fistulas. Presented at the 19th Annual Montefiore Medical 
Center Symposium on Current Critical Problems and New Techniques in Vascular Surgery, New York, NY, Nov 21, (1992).

[19] Queiroz, A. B, Mulatti, G. C, Aun, R, et al. Endovascular repair of a traumatic arteriovenous fistula involving the iliac bifurcation using an iliac branch device. J Vasc Surg. (2012). May;, 55(5), 1474-6.

[20] Hewitt, R L. and D J Collins Acute arteriovenous fistulas in war injuries. Ann Surg. (1969). March; , 169(3), 447-449.

[21] Spencer, T. A, Smyth, S. H, Wittich, G, et al. Delayed presentation of traumatic aortocaval fistula: A report of two cases and a review of the associated compensatory hemodynamic and structural changes. J Vasc Surg (2006). , 43, 836-40.

[22] Drapanas, T, Hewitt, R L, Weichert, R F, \& Rd, A. D Smith Civilian vascular injuries: a critical appraisal of three decades of management. Ann Surg. (1970). September; , 172(3), 351-360.

[23] Takenoshita, Y, Hasuoz, K, Matsushima, T, \& Oka, M. Carotid-Cavernous Sinus Fistula Accompanying Facial Trauma: Report of a Case with a Review of the Literature. J Cranio-Max Fac Surg. (1990). , 18, 41-5.

[24] Yu, P. T, Rice-townsend, S, Naheedy, J, Almodavar, H, \& Mooney, D. P. Delayed presentation of traumatic infrapopliteal arteriovenous fistula and pseudoaneursym in a year-old boy managed by coil embolization. J Pediatr Surg. (2012). Feb;47(2):e7-10., 10. 